\title{
Archaeal MCM Proteins as an Analog for the Eukaryotic Mcm2-7 Helicase to Reveal Essential Features of Structure and Function
}

\author{
Justin M. Miller and Eric J. Enemark \\ Department of Structural Biology, St. Jude Children's Research Hospital, 262 Danny Thomas Place, Memphis, TN 38105, USA
}

Correspondence should be addressed to Eric J. Enemark; eric.enemark@stjude.org

Received 27 January 2015; Accepted 5 April 2015

Academic Editor: Vickery L. Arcus

Copyright (c) 2015 J. M. Miller and E. J. Enemark. This is an open access article distributed under the Creative Commons Attribution License, which permits unrestricted use, distribution, and reproduction in any medium, provided the original work is properly cited.

In eukaryotes, the replicative helicase is the large multisubunit CMG complex consisting of the Mcm2-7 hexameric ring, Cdc45, and the tetrameric GINS complex. The Mcm2-7 ring assembles from six different, related proteins and forms the core of this complex. In archaea, a homologous MCM hexameric ring functions as the replicative helicase at the replication fork. Archaeal MCM proteins form thermostable homohexamers, facilitating their use as models of the eukaryotic Mcm2-7 helicase. Here we review archaeal MCM helicase structure and function and how the archaeal findings relate to the eukaryotic Mcm2-7 ring.

\section{Introduction}

The process of cell division requires precise duplication of genetic material. This duplication is carried out by replisomes that coordinate multiple protein activities to separate parental DNA strands and to synthesize new strands of complementary DNA. DNA strand separation and also the progression of the replisome along DNA are fueled by a replicative helicase. In eukaryotes, the replicative helicase is a large multiprotein complex, termed the CMG complex, which encircles leadingstrand DNA at the replication fork [1-3]. The CMG complex consists of $\mathrm{Cdc} 45$, the $\mathrm{Mcm} 2-7$ heterohexamer ring, and the GINS tetramer. The Mcm2-7 ring contains six unique gene products, which are loaded with Cdt1 at replication origins by the Origin Recognition Complex (ORC) and Cdc6 [4] to yield two Mcm2-7 rings that inactively encircle the DNA as a double-hexamer $[4,5]$ (Figure 1(a)). Helicase activation requires the Dbf4-dependent Cdc7 kinase (DDK) and cyclindependent kinases (CDKs) to phosphorylate $\mathrm{Mcm} 2-7$ and drive recruitment of Cdc45 and the GINS complex. Physical interaction of GINS and Cdc45 with phosphorylated Mcm27 supports formation of the active helicase complex [613]. Once activated, the two CMG complexes separate and translocate independently in opposite directions on opposing
ssDNA strands in the $3^{\prime} \rightarrow 5^{\prime}$ direction to generate two active replication forks $[3,12,14-18]$ (Figure 1(a)).

MCM proteins were first identified in a screen for genes essential for minichromosome maintenance in yeast [19]. The identified genes include 6 highly similar proteins with identifiable ATPase motifs [20, 21], Mcm2-7 (Figure 1(b)). The proposed function as the replicative helicase in eukaryotes was initially controversial because $\mathrm{Mcm} 2-7$ did not unwind DNA in vitro. However, this was resolved by observations that the larger CMG complex unwinds circular and forked doublestranded DNA substrates in vitro [2] and that the $S \mathrm{cMcm} 2-7$ complex can unwind DNA by itself when glutamate or acetate was included in the reaction buffer [1].

Electron microscopy studies have revealed general features of $\mathrm{Mcm} 2-7$ architecture, including a dynamic gap between the $\mathrm{Mcm} 2$ and $\mathrm{Mcm} 5$ subunits, an interface previously identified to serve as a "gate" in the ring $[1,22]$. EM studies have also shown that Cdc45 and the GINS tetramer associate with the Mcm2-7 hexamer near the $\mathrm{Mcm} 2 / 5$ gate $[23,24]$, potentially closing the gate (Figure $1(\mathrm{c})$ ). The CMG complex and the Mcm2-7 complex have not been visualized at atomic resolution since each complex has thus far resisted crystallization. A crystal to be used for structure determination must be well ordered, which requires all 


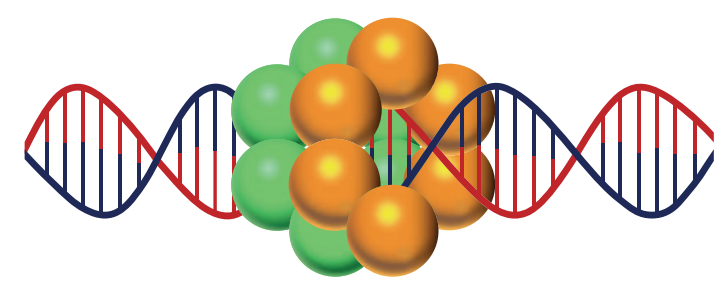

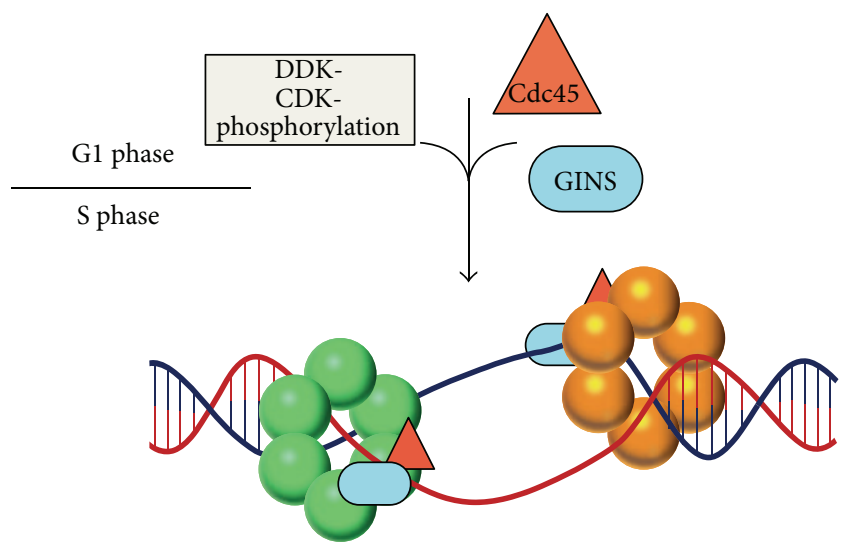

(a)

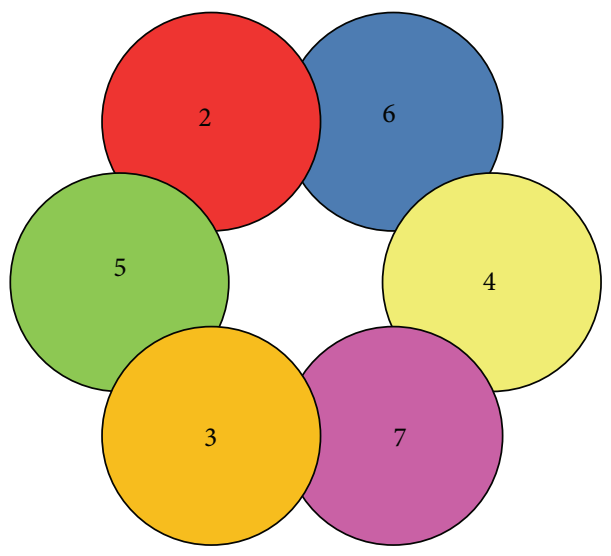

(b)

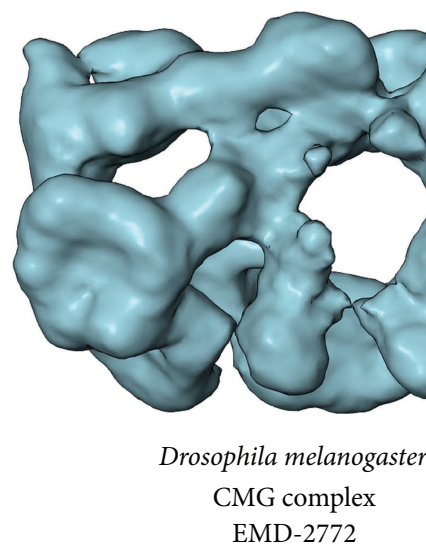

(c)

FIGURE 1: Mcm2-7 activation and organization. (a) Mcm2-7 rings are loaded as inactive double-hexamers at origins of replication by the Origin Recognition Complex (ORC), Cdc6, and Cdt1 (not shown). In a cell-cycle dependent fashion, the Dbf4-dependent Cdc7 kinase (DDK) and cyclin-dependent kinases (CDKs) drive the association of Cdc45 (red triangle) and the GINS complex (blue oval) with the phosphorylated Mcm2-7 ring to yield the active replicative helicase complex, termed the CMG complex (Cdc45-Mcm2-7-GINS). (b) Schematized view of the Mcm2-7 ring from the $\mathrm{N}$-terminal face with subunits labeled to illustrate the order of subunits around the ring $[65,86]$. The ring orientation has been selected to correspond with the orientation of the CMG complex shown in (c). (c) The three-dimensional electron microscopy reconstruction of the CMG complex illustrates basic architectural features. The CMG structure representation was prepared with the UCSF Chimera software package [87] and has been labeled with the Electron Microscopy Data Bank (EMDB) accession number.

related molecules to be consistently oriented throughout the crystal. A grossly symmetric ring, such as Mcm2-7, has special crystallization difficulties if the ring is able to adopt multiple orientations that are permuted. These will look similar at the macroscopic level but not at the atomic level. In contrast, archaeal MCM complexes often consist of six identical subunits, and thus all permutations are identical at the macroscopic and atomic level, potentially facilitating crystallization. Indeed, archaeal MCM complexes have been crystallized, which has led to atomic-level descriptions for major functions like DNA binding and ATP hydrolysis. Here we review archaeal MCM helicase structure and function with an emphasis on the advancements in our knowledge of the Mcm2-7 complex that have been derived from archaeal crystallographic studies.

\section{Archaeal MCM as a Model for Eukaryotic Mcm2-7}

As in eukaryotes, the replisomes of archaea centrally consist of a hexameric ring of MCM proteins that exhibits a $3^{\prime} \rightarrow 5^{\prime}$ polarity in in vitro double-stranded DNA 


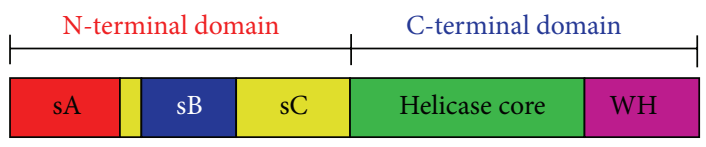

(a)

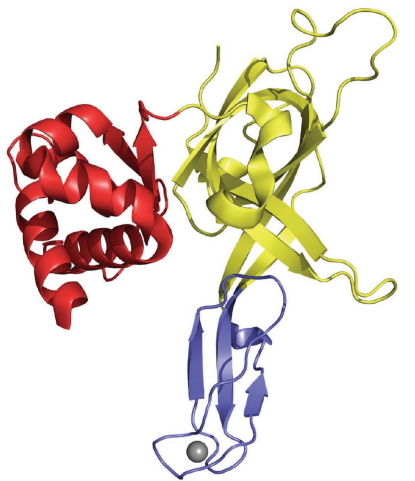

$M t \mathrm{MCM}_{\mathrm{N}}$

(1 subunit shown)

PDB: 1LTL

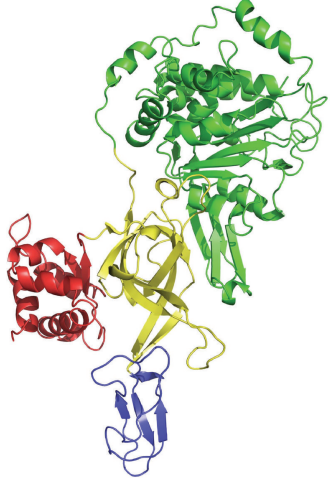

SsoMCM

PDB: 3F9V

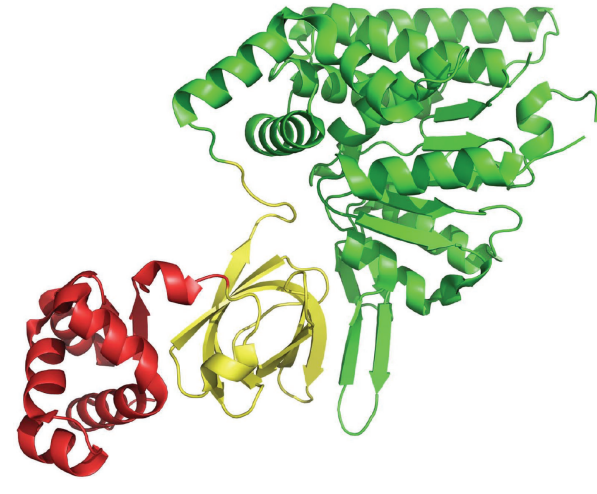

MkMCM2

PDB: 3F8T

(b)

FIGURE 2: General architecture of MCM proteins. (a) MCM monomers can be subdivided into N- and C-terminal tiers. Each tier can be further subdivided, where the N-terminal half includes subdomains A (red), B (blue), and C (yellow) and the C-terminal domain contains the helicase core (green) and a winged helix domain (magenta). (b) The crystal structures of one subunit of the Methanobacterium thermoautotrophicum MCM N-terminal domain double-hexamer $\left(M t \mathrm{MCM}_{\mathrm{N}}\right.$, PDB: 1LTL), monomeric Sulfolobus solfataricus MCM (SsoMCM, PDB: 3F9V), and monomeric Methanopyrus kandleri MCM2 (MkMCM2, PDB: 3F8T) each illustrate the general architecture with distinct subdomains A, B, C, and a AAA+ ATPase domain. Subdomains A, B, and C consist of a helical bundle (red), a zinc-binding domain (blue), and an oligonucleotide/oligosaccharide- (OB-) binding fold (yellow), respectively. The AAA+ domain architecture has five $\beta$-sheets flanked by $\alpha$ helices. All structure representations of Figure 2 were prepared with the Pymol software package [88].

(dsDNA) unwinding experiments [17, 25, 26]. The amino acid sequences of eukaryotic and archaeal MCM proteins are highly conserved. Due to the strong functional and sequence conservation, archaeal MCM proteins have proven to be powerful tools for elucidating essential features of MCM function. The MCM complexes of many archaea form homohexamers from a single gene product [27]. As such, these archaeal MCM complexes represent simplified versions of the eukaryotic Mcm2-7 complex and can serve as a model, both structurally and biochemically. These models have played a critical role in deciphering essential features of MCM structure and function because exclusively archaeal MCM complexes have generated crystal structures thus far. The identified essential features are likely to be conserved in all MCM complexes, including eukaryotic Mcm2-7.

\section{MCM Overall Architecture}

Based on electron microscopy studies, MCM hexamers form a ring with distinct $\mathrm{N}$-terminal and C-terminal tiers [25, 2831]. Both tiers can independently bind DNA with the $\mathrm{N}$ terminal tier showing a stronger affinity than the C-terminal tier [32]. The C-terminal tier contains the highly conserved $\mathrm{AAA}+$ (ATPases associated with various cellular activities) ATPase/helicase core [33-36] and a winged helix (WH) domain [37] (Figure 2(a)). The C-terminal ATPase tier alone from either SsoMCM [32] or ApeMCM [38] is sufficient for in vitro unwinding of dsDNA. The $\mathrm{N}$-terminal tier $\left(\mathrm{MCM}_{\mathrm{N}}\right)$ shows three consistent subdomains in crystal structures, $\mathrm{A}$ (sA), B (sB), and C (sC) [39-44] (Figures 2(a)-2(b)), as defined from the crystal structure of $M t \mathrm{MCM}_{\mathrm{N}}$ [39].

3.1. Subdomain A: Peripheral Helical Bundle. Subdomain A is a helical bundle located at the ring periphery and is directly connected to subdomain $\mathrm{C}$ by a short linker that may allow for dynamic interaction with the body of the protein [4548] (Figure 2(b)). Dynamic ring association could play a role in regulating $\mathrm{MCM}$ activities, perhaps by restricting access to protein interaction surfaces [44, 46, 49]. Deletion of subdomain A significantly reduces both single-stranded DNA (ssDNA) and dsDNA binding by MCM [28]. The $5^{\prime}-$ tail of Y-shaped DNA substrates has been proposed to wrap around the MCM exterior and fit into a binding pocket formed between solvent-exposed $\mathrm{C}$-terminal residues and subdomain A $[28,50-52]$. Hence, subdomain A orientation may impact protein:protein interactions, protein:DNA interactions, or both.

The mcm5-BOB1 P83L mutation in yeast allows cells to bypass the requirement for the S-phase activator protein Cdc7 [53]. The crystal structure of $M t \mathrm{MCM}_{\mathrm{N}}$ reveals that the corresponding residue sits at the center of the interface between subdomain A and subdomains B/C [39]. 
A "domain-push" model was proposed where mutation of the buried proline residue to an amino acid with a bulkier sidechain weakens the interaction between subdomain $\mathrm{A}$ and subdomains $\mathrm{B} / \mathrm{C}$. This hypothesis was tested by introducing P83L, P83W, or P83K Mcm5 mutations in yeast cells [39]. Consistent with the "domain-push" model, bulky side-chains (P83L, P83W, or P83K) allowed S-phase checkpoint bypass, but P83G Mcm5 mutant-containing cells behaved equivalent to wild-type cells. The N-terminal subdomain A has most often been observed in EM and crystal structures to pack against subdomains B/C [39, 40, 43, 45, 48], but subdomain A has also been observed in a different conformation that is extended away from the body of the protein $[44,45,54]$. The "domain-push" may expose a protein interaction surface that is normally only exposed following DDK phosphorylation. Thus, the observation that the $m c m 5-B O B 1$ mutant is able to bypass the $S$-phase checkpoint may be a result of a shift in protein interaction partners.

3.2. Subdomain B: Zinc-Binding Domain. All X-ray crystal structures of $\mathrm{MCM}_{\mathrm{N}}$ reveal a tetrahedrally coordinated zinc ion bound to subdomain B [39, 40, 43] (Figure 3(a)). Critical zinc-binding amino acid side-chains are located on three antiparallel $\beta$-strands [39-41, 43, 44]. MCM biochemical activities show extreme sensitivity to mutation of the zincbinding residues, and a cysteine to serine point mutation in $M t \mathrm{MCM}$ ablates dsDNA unwinding, DNA-dependent ATPase activity, and ssDNA binding [55]. The zinc-binding sequence motif is commonly $\mathrm{CX}_{2} \mathrm{CX}_{n} \mathrm{CX}_{2} \mathrm{C}\left(\mathrm{C}_{4}\right.$ type) [39, 43], although a histidine residue is also possible, as observed in the crystal structure of SsoMCM $_{\mathrm{N}}$ (Figures 3(a)-3(b)) [40] and in the sequence of ApeMCM (Figure 3(b)). Other than $\mathrm{Mcm} 3$, all of the subunits of eukaryotic $\mathrm{Mcm} 2-7$ possess four highly conserved cysteines that likely coordinate a zinc ion with a tetrahedral geometry. The spacing of the cysteines in these $\mathrm{Zn}$-binding sequence motifs show family-specific patterns (Figure 3(b)). Mcm2 has a $\mathrm{CX}_{2} \mathrm{CX}_{n} \mathrm{CX}_{2} \mathrm{C}$ motif analogous to those of $M t \mathrm{MCM}$ and PfMCM. Moreover, a highly similar $\mathrm{CX}_{2} \mathrm{CX}_{n} \mathrm{CX}_{2-4} \mathrm{C}$ motif of $\mathrm{Mcm} 4, \mathrm{Mcm} 6$, and $\mathrm{Mcm} 7$ also likely binds a zinc ion $[55,56]$. In contrast, the four conserved cysteines of $\mathrm{Mcm} 5$ have an unusually large spacing between the third and fourth cysteine. The familyspecific conservation of the zinc-binding motif, including the lack of an obvious motif in $\mathrm{Mcm} 3$, suggests that the zincbinding sites provide an important mechanism for regulation of eukaryotic Mcm2-7 activities.

3.3. Subdomain $C$ : $O B$-Fold. Subdomain $C$ has an oligonucleotide/oligosaccharide fold (OB-fold), a fold associated with DNA binding [57] (Figure 2(b)). Multiple residues in subdomain $\mathrm{C}$ are critical for DNA binding and hexamerization $[41,43,49,58]$. Further, $\beta$-loop motifs found in subdomain $\mathrm{C}$ appear to facilitate allosteric communication between the $\mathrm{N}$ - and C-terminal tiers [59, 60]. A phenylalanine to isoleucine mutation (F345I) in mouse Mcm4 subdomain C has been termed Chaos3 (chromosome aberrations occurring spontaneously 3) [61]. Homozygous $\mathrm{Mcm} 44^{\text {Chaos3/Chaos3 }}$ mice develop mammary adenocarcinomas within an average of 12 months for $>80 \%$ of cases, and mouse cells that possess only F345I mutant Mcm4 (either Mcm4 $4^{\text {Chaos3/- }}$ or $\mathrm{Mcm} 4^{\text {Chaos3/Chaos3 }}$ ) exhibit hypersensitivity to chromosomal breakage [61]. Strains of Saccharomyces cerevisiae with the analogous mutation ( $\mathrm{ScMcm} 4 \mathrm{F391I}$ ) exhibit the minichromosome loss phenotype typically associated with a lossof-function for $\mathrm{Mcm} 2-7$ [61]. The phenylalanine residue involved is highly conserved as an aromatic residue in all eukaryotic Mcm2-7 subunits and also in $M t \mathrm{MCM}$ ( $M t \mathrm{MCM}$ F170) [61]. The highly conserved aromatic residue is at the center of a hydrophobic intersubunit interface in the structure of $M t \mathrm{MCM}$ [39] and is therefore likely critical to the structural integrity of the MCM ring. Although the Chaos3 mutation may not completely prevent interaction of $\mathrm{Mcm} 4$ with other $\mathrm{Mcm} 2-7$ subunits, the mutation could alter the intersubunit orientations to disrupt DNA binding by the MCM single-stranded binding motif (MSSB, described further below) [43] to generate genomic instability, a general hallmark of cancer cells [62-64].

3.4. AAA+ATPase Core Domain. Crystal structures of MCM proteins containing both $\mathrm{N}$ - and C-terminal tiers have revealed a canonical AAA + ATP binding and hydrolysis site in the C-terminal tier. The MCM AAA+ domain has 5 parallel $\beta$-strands flanked on either side by $\alpha$-helices [41, 42, 44] (Figure 2(b)). Consistent with other AAA + family members, such as domain 2 of $\mathrm{N}$-ethylmaleimide sensitive fusion protein, the strand order is $\beta 5-\beta 1-\beta 4-\beta 3-\beta 2$, which strictly positions key catalytic residues to generate a competent ATPase site $[34,41,42,44]$. The MCM AAA+ ATP binding and hydrolysis site contains canonical cis- and trans-acting elements like the cis-acting Walker A and Walker B motifs and the trans-acting "SRF" arginine finger motif $[65,66]$. Mutation of nearly any residue of the MCM AAA+ active abolishes ATPase activity $[32,66,67]$.

3.5. Winged Helix-Turn-Helix Domain. Amino acid residues found at the extreme C-terminus of archaeal MCM proteins [37], Mcm6 [68], and the replication factor Mcm10 [69, 70] are predicted to adopt a flexible winged helix-turn-helix fold (Figure 2(a)). For most archaeal MCM proteins, this represents the C-terminal 60-70 amino acids. The NMR structure of the Mcm6 winged helix (WH) domain indicates that the isolated domain is well ordered $[68,71]$. However, it is connected to the core of the protein via a flexible linker that allows for mobility of the domain similar to subdomain A [48]. Consistent with this, all X-ray crystal structures containing the C-terminal tier reported to date have been with constructs that lack the WH domain, suggesting that the orientation of this domain is intrinsically flexible [41, 42, 44]. The deletion of the WH region in SsoMCM or MtMCM results in a nearly 2-fold enhancement in ATPase activity $[32,72]$. For SsoMCM, deletion of the WH domain also yields a 15-fold increase in dsDNA unwinding activity [32]. Taken together, the results suggest that the WH domain does not play an essential role during normal unwinding and may instead function during the initial assembly or activation of the helicase or to sense atypical DNA structures, such as damaged DNA, and to signal their presence. Consistent with 


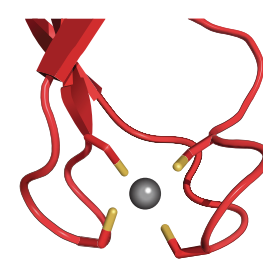

$M t \mathrm{MCM}_{\mathrm{N}}$

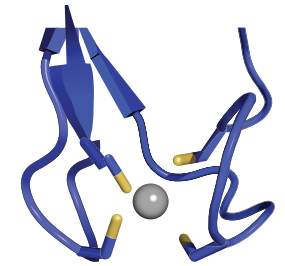

Pf $\mathrm{MCM}_{\mathrm{N}}$ PDB: 4POG

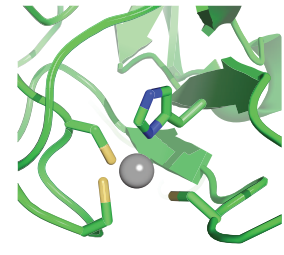

SsoMCM PDB: 2VL6

(a)

O $\bigcirc$

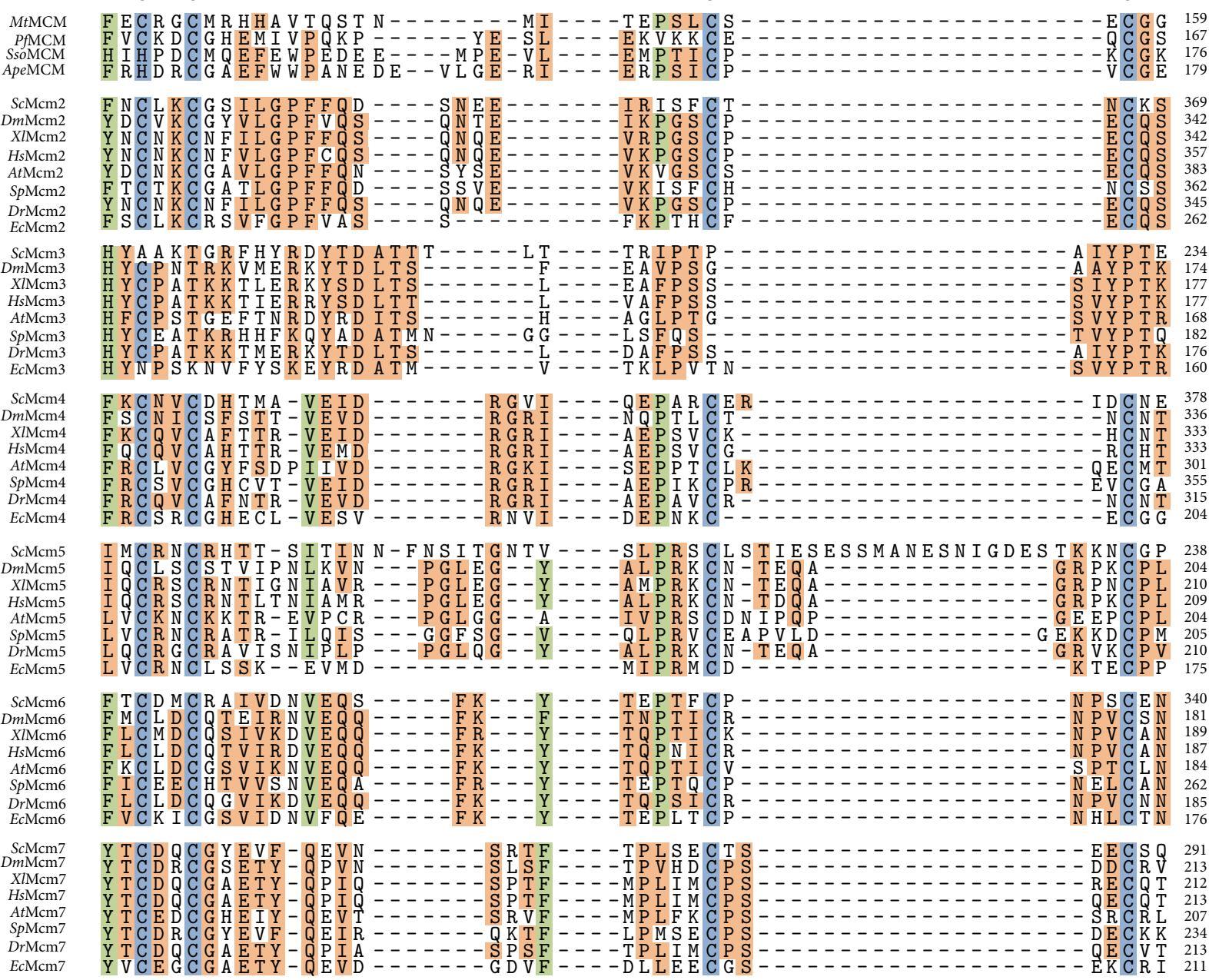

(b)

FIGURE 3: Subdomain B contains a zinc-binding motif. (a) All $\mathrm{MCM}_{\mathrm{N}} \mathrm{X}$-ray crystal structures contain a zinc atom bound with a tetrahedral geometry. Each structure is depicted in a cartoon representation with zinc-binding side-chains shown in stick. Cysteine sulfurs are colored in yellow, and histidine nitrogen atoms are colored in blue. The remainder of each protein is shown as red, blue, and green for $M t \mathrm{MCM}_{\mathrm{N}}$ (PDB: 1LTL), Pf $\mathrm{MCM}_{\mathrm{N}}$ (PDB: 4POG), and $S$ soMCM $\mathrm{N}_{\mathrm{N}}$ (PDB: 2VL6), respectively. Zinc atoms are shown as gray spheres. All structure representations of Figure 3 were prepared with the Pymol software package [88]. (b) A multiple sequence alignment shows the strong sequence conservation of the MCM zinc-binding domain. The spacing of the cysteine residues in MCM Zn-binding sequence motifs shows familyspecific patterns, where residues displaying a high degree of sequence conservation across all MCM proteins or family-specific sequence conservation have been colored as either green or orange, respectively. Residues involved in zinc-binding are highlighted in blue with a blue dot for emphasis. Mcm3 does not possess an obvious zinc-binding motif, and Mcm5 has an abnormally large spacing between the third and fourth cysteine residues. 

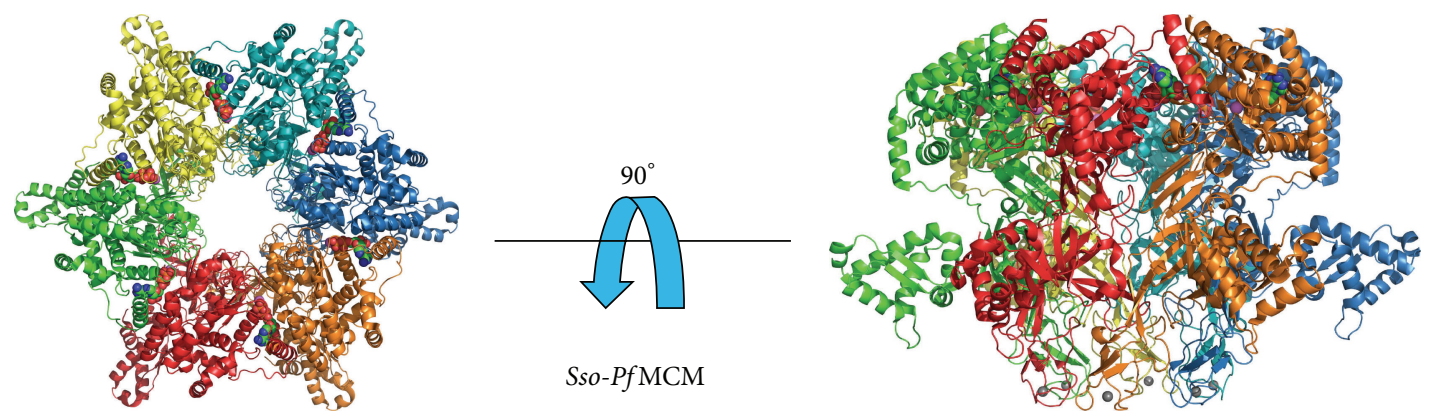

PDB: 4R7Y

(a)

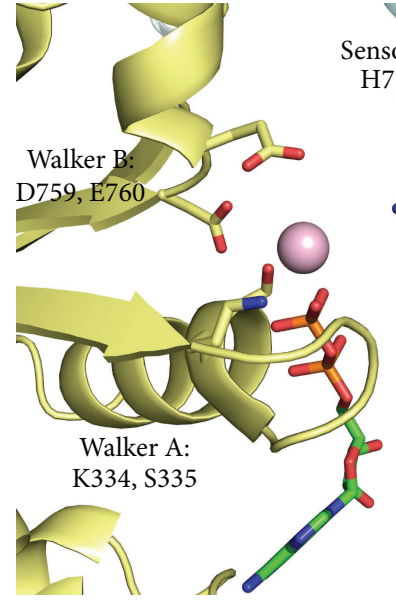

(b)

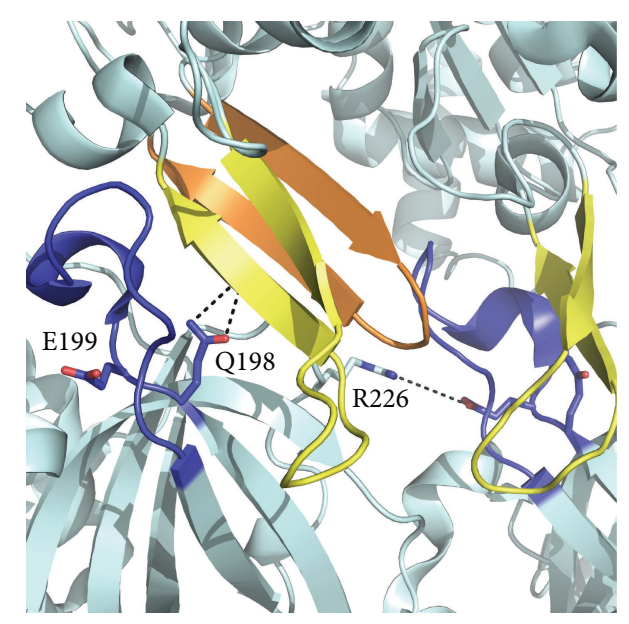

(c)

FIGURE 4: Crystal structure of a nearly full length MCM hexamer. An ADP-bound, nearly full length MCM protein was crystallized by creating a chimeric fusion protein of the N-terminal domain of SsoMCM and the C-terminal tier of PfMCM. (a) Views of the Sso-PfMCM hexamer crystal structure parallel and perpendicular to the central channel with each subunit uniquely colored. Magnesium ions are shown as magenta spheres, ADP molecules are shown in space-filling view, and zinc ions are shown as gray spheres. In the view parallel to the central channel, the ATPase domains are projected out of the page. In the perpendicular view, the ATPase domains are located at the top and the $\mathrm{N}$-terminal domains are located on the bottom. (b) The ATPase site is formed at subunit interfaces by cis-and trans-acting residues shown in stick and labeled. The Walker A and Walker B residues of one subunit are positioned at the left side of the site (yellow), while three basic residues are located on the right side of the site (blue). The bound ADP molecule is shown in stick, and the magnesium ion is represented as a magenta sphere. (c) Residues of the allosteric communication loop (ACL) interact across subunit interfaces and also with main-chain atoms of the helix-2-insert (h2i) $\beta$-hairpin motif. The ACL is shown in blue and the h2i and presensor-1- $\beta$-hairpin (ps1 $\beta$ ) are shown in yellow and orange, respectively. Dashed lines indicate the discussed molecular interactions. All structure representations of Figure 4 were prepared with the Pymol software package [88] and PDB accession code 4R7Y.

such a role, this domain is expected to sit directly proximal to the entering dsDNA to be unwound [73].

\section{MCM Hexamer}

Although archaeal MCM proteins are often robust hexamers in solution [32, 66, 73-75], they have proven to be highly resistant to crystallization in a hexameric form. This challenge was overcome by a chimeric fusion of the $\mathrm{N}$-terminal domain of SsoMCM with the ATPase domain of Pf MCM (Sso-PfMCM), which crystallized as a hexamer bound to Mg:ADP [44]. In the Sso-PfMCM hexamer structure, the $\mathrm{N}$ - and C-terminal domains form distinct tiers stacked on top of one another (Figure 4(a)). The cocrystallized ADP revealed the general architecture of the AAA+ ATP binding site and the specific interactions between the protein and bound nucleotide (Figure 4(b)). A "sensor 3" residue was identified based on the observation of a histidine side-chain that projects into the active site similar to sensor 2 and the arginine finger. The structure also revealed atomic details of the intermolecular interactions between the subunits and also intramolecular interactions between the tiers (Figure 4(c)). In addition, the Sso-PfMCM structure crystallized with subdomain $\mathrm{A}$ in an extended conformation, in contrast to the previous $\mathrm{N}$-terminal MCM crystal structures with subdomain A packed against subdomains $\mathrm{B} / \mathrm{C}$, consistent with an ability for subdomain A to adopt multiple discrete conformations. 


\section{ATPase Site}

Consistent with other AAA+ superfamily members, the MCM ATP binding and hydrolysis site is at the interface of two adjacent subunits with catalytic residues contributed both in cis and in trans. The site is competent to catalyze ATP hydrolysis only when all requisite residues are precisely arranged. In the ADP-bound Sso-Pf MCM crystal structure, Walker A residue K334 is observed to project into the ATP binding site, and residue S335 coordinates a magnesium cation (Figure 4(b)) [44]. Based on previously proposed $\mathrm{AAA}+$ ATPase catalytic mechanisms, this magnesium cation likely positions the $\beta$ - and $\gamma$-phosphates of the bound ATP molecule [76] and neutralizes an accumulated negative charge on the $\gamma$-phosphate during hydrolysis $[34,76]$. Based on crystal structures of other AAA+ family members, the second conserved acidic residue of the Walker $B$ motif (E760) is the catalytic base for a water molecule to perform a nucleophilic attack on the $\gamma$-phosphate of the bound ATP.

The trans-acting elements of AAA+ ATPase sites occupy different positions depending on whether ATP, ADP, or no nucleotide is bound to communicate the active site status to the rest of the protein $[66,77,78]$. The trans-acting residues must be able to reach the ATP molecule to generate a hydrolysis site with the adjacent subunit. The subunit interface is likely dynamic because the positions of the transacting residues in the ADP-bound hexameric Sso-Pf MCM structure are too distant for such interaction, and the subunit interface therefore needs to constrict in order for the transacting residues to interact with ATP $[44,77]$. Trans-acting MCM residues include "sensor 2" [44, 66, 79], the "arginine finger" $[44,66]$, and "sensor 3" [44]. The arginine finger may function to polarize the $\gamma$-phosphate during ATP hydrolysis [76]. The trans-acting "sensor 2" element of MCM contrasts most AAA+ ATPases that typically use this motif as a cisacting residue $[42,44,66,79]$.

\section{MCM Double-Hexamer}

In eukaryotes, two Mcm2-7 rings are initially loaded as an inactive double-hexamer at replication origins $[4,5]$. The $\mathrm{X}$ ray crystal structure of $M t \mathrm{MCM}_{\mathrm{N}}$ revealed an MCM doublehexamer with two hexamers arranged head to head via subdomain B domain interactions (Figure 5) [39], providing a model for the Mcm2-7 double-hexamer interaction. A single arginine to alanine mutation at the $M t \mathrm{MCM}_{\mathrm{N}}$ hexamer:hexamer interface disrupted the interaction in favor of single hexamers [80]. A double-hexamer structure had previously been observed for full length $M t \mathrm{MCM}$ in scanning transmission electron microscopy images [25]. In contrast to the stable double-hexamer of $M t \mathrm{MCM}, \mathrm{X}$-ray crystal structures of $S$ so $\mathrm{MCM}_{\mathrm{N}}$ and $P f \mathrm{MCM}_{\mathrm{N}}$ consist of single hexamers $[40,43]$. Although a double-hexamer structure has not yet been universally detected among archaeal MCM complexes, double-hexamer architecture is likely to be conserved when MCM rings are first loaded onto DNA prior to the onset of bidirectional replication [81].

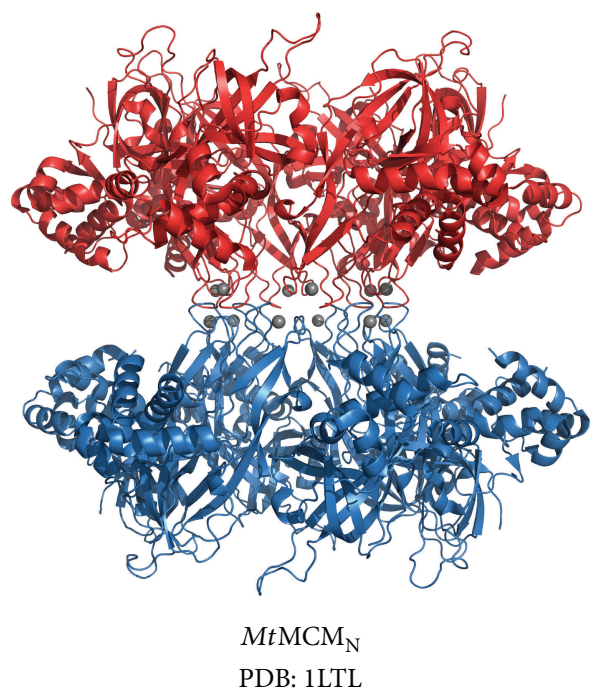

Figure 5: $M t \mathrm{MCM}_{\mathrm{N}}$ double-hexamer crystal structure. The $M t \mathrm{MCM}_{\mathrm{N}}$ double-hexamer crystal structure is shown in cartoon representation with one hexamer colored red and the other blue. The two hexamers interact head to head via subdomain B interactions. Zinc atoms are shown as gray spheres. The figure was prepared with the Pymol software package [88] and PDB accession code 1LTL.

\section{DNA Binding}

The N-terminal and C-terminal domains of MCM are independently able to bind DNA with several modules that line the interior channel of the hexameric ring. These modules and their interaction with DNA are detailed further below.

7.1. MCM Single-Stranded Binding Motif (MSSB). The MCM $\mathrm{N}$-terminal domain binds ssDNA as revealed by a recent X-ray crystal structure of $P f \mathrm{MCM}_{\mathrm{N}}$ bound to ssDNA [43]. In this structure, ssDNA binds in the plane of the ring, rather than perpendicular as would be expected if DNA were threaded through the central channel (Figure 6(a)) [43]. This DNA binding configuration contrasts the configurations previously seen for the nucleic acid complexes of the hexameric helicases E1 [77], DnaB [82], and Rho [83] where nucleic acid binds perpendicular to the plane of the hexamer. When viewed from the $\mathrm{N}$-terminal side of the complex (Figure 6(a)), the ssDNA binds with $3^{\prime}$ to $5^{\prime}$ polarity in the clockwise direction around the ring. The $\mathrm{MCM}_{\mathrm{N}}$ :ssDNA structure revealed that 4 ssDNA nucleotides are bound per subunit, which also contrasts with other hexameric helicases that bind either one (E1 [77] and Rho [83]) or two (DnaB [82]) nucleotides per subunit. Interestingly, the $\mathrm{MCM}_{\mathrm{N}}$ :ssDNA structure reveals that ssDNA is bound across subunit interfaces but is not bound at all interfaces of a hexamer (Figure 6(b)) [43]. The intersubunit distance appears to dictate whether ssDNA can bind at a particular subunit interface with ssDNA observed only at tighter interfaces [43].

The $P f \mathrm{MCM}_{\mathrm{N}}$ :ssDNA cocrystal structure revealed specific amino acid residues of the OB-fold subdomain $\mathrm{C}$ that 


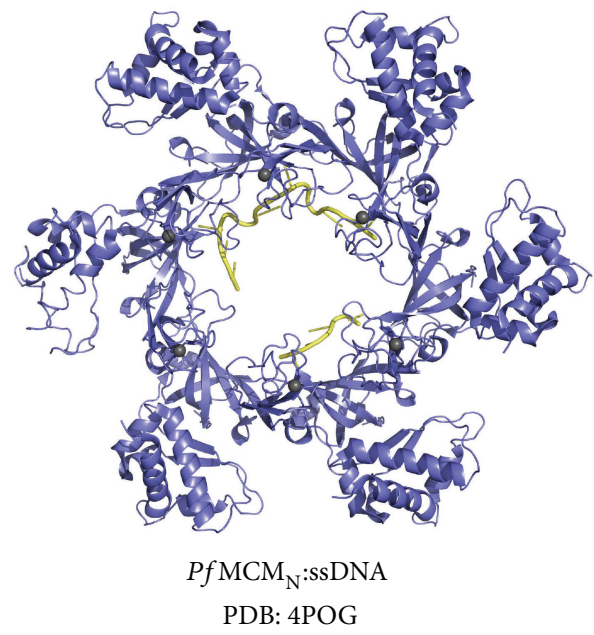

(a)

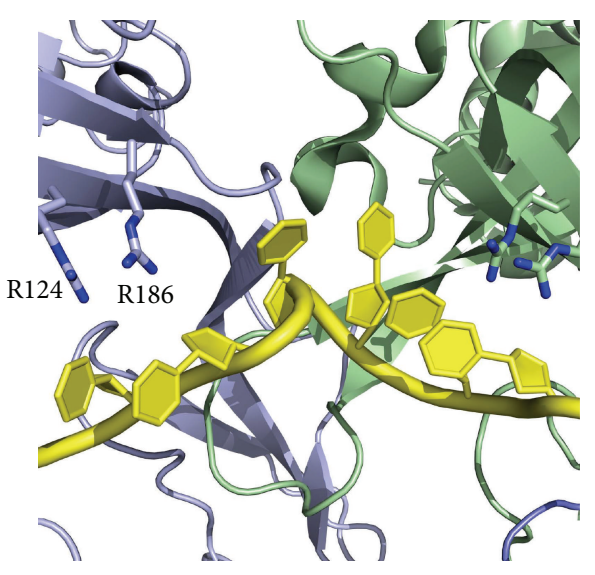

(b)

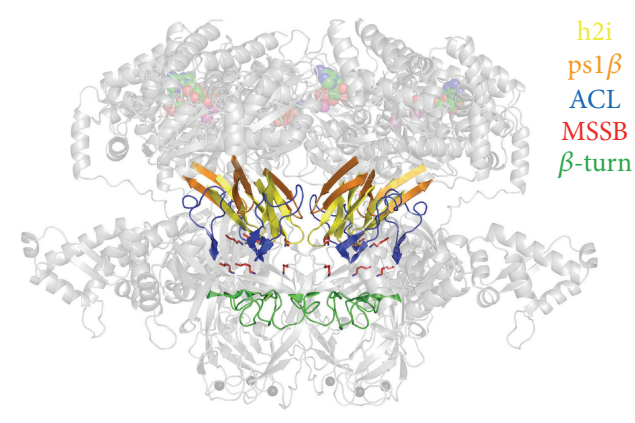

Sso-Pf MCM

PDB: 4R7Y

(c)

FIGURE 6: Features of DNA binding in MCM hexamers. (a) The cocrystal structure of $P f \mathrm{MCM}_{\mathrm{N}}$ bound to ssDNA revealed that DNA binds in the plane of the hexamer. The DNA binds with a $3^{\prime}$ to $5^{\prime}$ polarity in the clockwise direction around the ring when viewed from the Nterminal side of the hexamer. DNA is shown in yellow and zinc ions are shown as grey spheres. (b) DNA binds across subunit interfaces

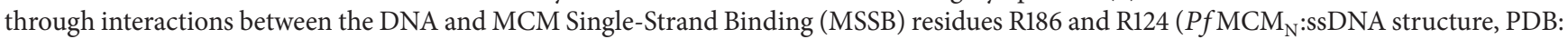
4POG), shown in stick. (c) Important residues of the central channel in the MCM hexamer structure (PDB: 4R7Y) with other components transparent. The h2i, ps1 $\beta$, ACL, MSSB, and N-terminal $\beta$-turn motifs are shown in yellow, orange, blue, red, and green, respectively. All structure representations of Figure 6 were prepared with the Pymol software package [88].

bind to ssDNA (Figure 6(b)). This motif is termed the MCM Single-Strand Binding (MSSB) motif (Figure 6(b)) and forms a positively charged DNA binding pocket near the interface of the N- and C-terminal tiers (Figure 6(c)). Residues R124 and R186 were demonstrated to most significantly impair DNA binding where the affinity of R124A and R186A point mutants for ssDNA decreased 7- and 6-fold, respectively [43]. The R124A/R186A double-mutant exhibits a 25 -fold reduction in ssDNA binding affinity with much higher protein concentrations required for observation of binding relative to the wild-type protein. Mutation of similarly positioned residues in SsoMCM abolishes dsDNA unwinding activity [74].

In Saccharomyces cerevisiae, the Pf MCM R124 and R186 MSSB residues are conserved as either an arginine or lysine in Mcm4, Mcm6, and Mcm7 [43]. In Mcm2, Mcm3, and Mcm5, only one of the two positively charged residues is conserved but not both. Interestingly, complementation studies in yeast demonstrated that while single-subunit MSSB mutants are viable, cells with two-subunit MSSB mutations are lethal: $\mathrm{Mcm} 4 / \mathrm{Mcm} 6, \mathrm{Mcm} 4 / \mathrm{Mcm} 7$, and $\mathrm{Mcm} 6 / \mathrm{Mcm} 7$ [43] (Figure 1(b)). In fact, any of these combinations leave the Mcm2-7 ring defective in helicase loading and severely defective for replication [43]. These data suggest a role for the MSSB during Mcm2-7 helicase loading and activation.

7.2. N-Terminal $\beta$-Turn. A second $\mathrm{N}$-terminal motif implicated in DNA interaction is a $\beta$-turn that projects into the central channel of the hexamer (Figure 6(c)). In SsoMCM, this motif spans residues 241-251 (polypeptide sequence QDSPVKRGSRA) between $\beta$-strands $\beta 11$ and $\beta 12$ [40]. The $\beta$-turn is present in all archaeal MCM proteins and has been studied in ScMcm4 and ScMcm5 [39, 40, 43, 84]. The length and sequences of this loop vary among Mcm27 subunits. In X-ray crystal structures of archaeal MCM 
hexamers, the $\mathrm{N}$-terminal $\beta$-turn is the narrowest part of the central channel, varying from $17 \AA$ to $23 \AA[39,40$, 43]. Despite poor sequence conservation in this region, the presence of positively charged residues on the $\beta$-turn is required for DNA binding $[39,73]$. Double-mutation of two positively charged residues (K246A/R247A) on the tip of the N-terminal $\beta$-turn in SsoMCM leads to an 8-fold reduction in DNA binding [73]. Similarly, DNA binding is abolished for the comparable alanine double-mutant of MtMCM (R226A/228A) [39]. Based on these experiments, the DNA binding activity attributed to the $\mathrm{N}$-terminal $\beta$-turn is proposed to play a critical role in DNA loading $[73,84]$. Structure-guided yeast genetic approaches have suggested that the $\beta$-turn of $S c M c m 5$ is important for binding to origins of replication and subsequent initiation of DNA replication [84]. Thus, the $\mathrm{N}$-terminal $\beta$-turn likely has a major role in facilitating initial DNA binding.

7.3. Presensor-1 $\beta$-Hairpin ( $p$ s1 $\beta$ ) and Helix-2-Insert (h2i). The ATPase domain of each MCM monomer contains two $\beta$-hairpin motifs that project into the central channel of the hexamer, the helix-2-insert (h2i), and the presensor-1- $\beta$ hairpin (ps1 $\beta$ ) (Figures $7(\mathrm{a})-7(\mathrm{~b}))[41,42,44,72]$. The h2i motif is defined by a $\beta-\alpha-\beta$ insertion in primary sequence between Walker A and Walker B motifs $[35,72,79]$. This feature is found as a unique insert within helix-2 of the conserved ASCE (additional strand catalytic glutamate) ATPase family $[35,72,85]$. Similarly, the psl $\beta$ feature represents an insertion between sensor 1 motif and the preceding helix [35]. Within the AAA+ superfamily, the ps $1 \beta$ superclade includes the SFIII helicases, HslU, ClpX, Lon, ClpA, MCM, dynein, midasin, YifB, and many others [35]. The ps1 $\beta$ superclade is subdivided into HslU/ClpX/Lon/ClpAB-C and h2i subclades, where MCM proteins belong to the h2i subclade since they contain both the ps1 $\beta$ and the h2i motifs [35].

MCM mutants lacking either the ps1 $\beta$ or h2i motifs are unable to catalyze dsDNA unwinding, despite still being competent for DNA binding [72, 73]. The psl $\beta$-deletion mutant displays a weakened binding affinity for Y-shaped DNA of $343 \mathrm{nM}$ versus $152 \mathrm{nM}$ for wild-type protein [73]. Interestingly, this contrasts an h2i-deletion mutant, which displays a shift in binding affinity for a blunt dsDNA substrate from greater than $1 \mu \mathrm{M}$ for the wild-type protein to $\sim 8 \mathrm{nM}$ for the h2i-deleted mutant [72]. The observation of tighter binding for an h2i-deleted MCM protein may suggest a role for the $\mathrm{h} 2 \mathrm{i}$ in destabilizing DNA:protein interactions, which could facilitate the movement of DNA during unwinding. The primary role of the psi $\beta$ motif is most likely to bind DNA since deletion of the $\operatorname{psl} \beta$ feature yields the weakened affinity expected for a motif intimately involved in DNA binding. The lack of detectable unwinding activity for either the psi $\beta$ or h2i-deletion mutants indicates that both C-terminal $\beta$ hairpins are essential.

\section{DNA Translocation}

The conformation of the h2i and ps1 $\beta$ motifs has been proposed to depend on whether ATP or ADP is bound [41, $42,72]$. In the ADP-bound MCM hexamer structure, these loops are observed to be in a "down" position (Figure 7(a)) [44]. Thus, a view perpendicular to the central channel of the hexamer defines the C-terminal domain face as the "up" direction and the N-terminal face as the "down" direction. The orientation of the MCM hexamer shown in Figure 7(a) would result in DNA being translocated with a net movement from the top to the bottom face. Consequently, this motion implies that the h2i and ps1 $\beta$ motifs would be in the "up" position in the ATP-bound state. Following hydrolysis of ATP to ADP, the loops would move to the "down" position shown in Figure 7(a). This direction of DNA translocation is consistent with earlier FRET studies showing that MCM hexamers bind forked DNA with the C-terminus facing the leading direction [73].

The projection of each $\mathrm{C}$-terminal $\beta$-hairpin into the central channel of the MCM hexamer is expected to position positively charged residues for interaction with DNA (Figure 7(b)). This is conceptually similar to other hexameric helicases such as E1 [77], DnaB [82], and Rho [83]. In the crystal structures of each of these hexameric helicases, positive residues on loops projected into the central channel interact with nucleic acid in a spiral-staircase-like arrangement (Figures $7(\mathrm{c})-7(\mathrm{~h})$ ). In this mode of binding, the central channel loops from adjacent subunits occupy different heights around the ring to form a "spiral-staircase" shape. The movement of DNA is then achieved by the concerted movement of the entire staircase of loops [77, 82, 83]. As an example, the adjacent subunits of the E1 structure adopt ATPlike, ADP-like, and apo forms around the ring that clearly demonstrate that the loop positions depend on whether ATP, ADP, or no nucleotide is bound at the associated ATP binding site [77]. Basic features of this mode of DNA binding and translocation are most likely shared by all the hexameric helicase superfamilies: SFIII (E1), SFIV (DnaB), SFV (Rho), and SFVI (MCM) [89].

Although MCM proteins display high homology with SFIII helicases, the presence of both the $h 2 i$ and $p s 1 \beta$ features in MCM proteins suggests significantly different mechanisms for coupling the energy of ATP binding and hydrolysis to dsDNA unwinding. Deletion of the h2i $\beta$-hairpin in MtMCM results in $\sim 12$-fold increase in dsDNA-stimulated ATPase activity relative to wild-type $M t \mathrm{MCM}$ [72]. However, no detectable dsDNA unwinding is observed for the h2i-deletion mutant, despite the observation that the h2i-deleted mutant still binds both ssDNA and dsDNA [72]. Taken together, these observations suggest that the deletion of the h2i motif actually disrupts the coupling of ATP binding and hydrolysis to DNA unwinding such that the helicase can bind dsDNA and also hydrolyze ATP but cannot unwind dsDNA. This is conceptually analogous to an automobile engine shifted to neutral with deletion of the h2i motif disengaging the MCM motor from dsDNA unwinding.

8.1. Allosteric Communication Loop. While the C-terminal helicase core can catalyze unwinding of dsDNA in the absence of the MCM N-terminal tier, inclusion of the Nterminal tier results in an increase in the processivity of MCM catalyzed dsDNA unwinding [32]. Such increases in processivity are observed whether the two halves are expressed as 


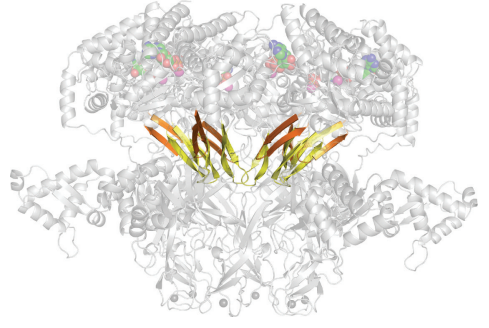

MCM (PDB: 4R7Y)

(a)

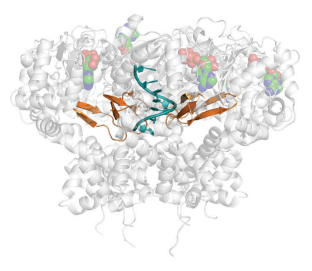

E1 (PDB: 2XGA)

(c)

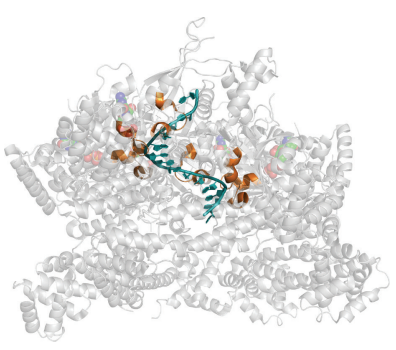

DnaB (PDB: 4ESV)

(e)

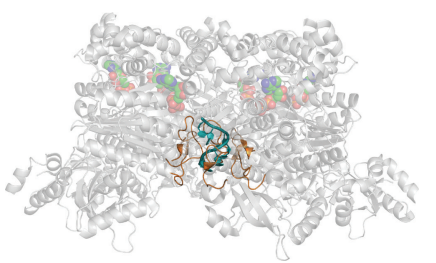

Rho (PDB: 3ICE)

(g)

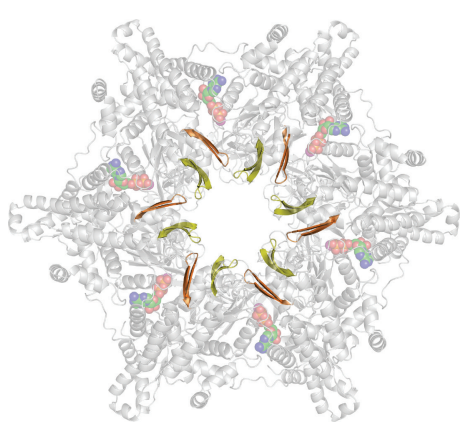

MCM (PDB: 4R7Y)

(b)

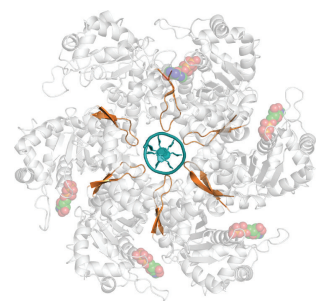

E1 (PDB: 2XGA)

(d)

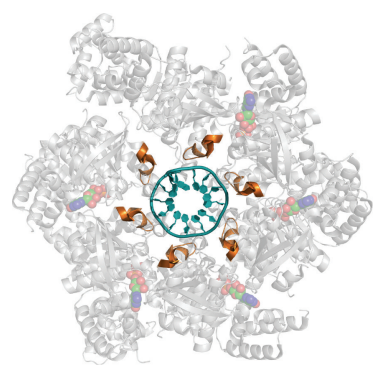

DnaB (PDB: 4ESV)

(f)

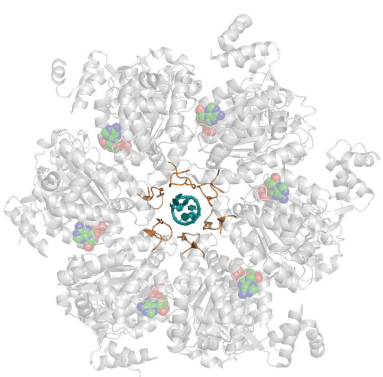

Rho (PDB: 3ICE)

(h)

Figure 7: Comparison of MCM with representative members of other hexameric helicase superfamilies. The hexameric MCM structure viewed either perpendicular (a) or parallel (b) to the central channel axis. The MCM h2i and ps1 $\beta$ central channel modules are colored yellow and orange, respectively. (c-h) Comparison with representative members of other helicase superfamilies such as E1 ((c-d), SFIII, PDB: 2XGA), DnaB ((e-f), SFIV, PDB: 4ESV), and Rho ((g-h), SFV, PDB: 3ICE) suggests that MCM central channel modules will move "up" and "down" to translocate DNA. In the crystal structures of each of these hexameric helicases, basic residues on loops projected into the central channel interact with nucleic acid in a spiral-staircase-like arrangement. In this mode of binding, the central channel loops from adjacent subunits occupy different heights around the ring to form a "spiral-staircase" shape. The movement of DNA is then achieved by the movement of the central channel loops. DNA is colored in teal and DNA binding loops are colored orange. All structures are viewed either perpendicular (left column) or parallel (right column) to the central channel axis. All structure representations of Figure 7 were prepared with the Pymol software package [88]. 
a single gene product or when each half is expressed separately and incubated together during unwinding experiments [32]. An important interaction between each half is mediated through a conserved loop found at the interface of the $\mathrm{N}$ - and C-terminal tiers termed the "allosteric communication loop" (ACL, Figure 6(c)). Mutational studies have revealed that point mutations in this loop can have either stimulatory or inhibitory effects on the observed MCM dsDNA unwinding activity [60]. For the majority of point mutations introduced to this loop, the rate of $M t \mathrm{MCM}$ catalyzed dsDNA unwinding is observed to decrease relative to the wild-type protein, but mutation of either Q181 or E185 to alanine actually results in an increase in unwinding rate. Deletion of the entire ACL causes a loss of unwinding activity. However, activity is restored by the additional deletion of the $\mathrm{N}$-terminal $\beta$ turn, which suggests the position of the $\mathrm{N}$-terminal $\beta$-turn could be controlled by the ATP hydrolysis cycle via the ACL [59]. In the Sso-PfMCM hexamer crystal structure, a fully conserved glutamine residue (homologous to MtMCM Q181) at the C-terminal end of the ACL interacts with the h2i $\beta$-hairpin of the C-terminal AAA+domain (Figure 4(c)). This glutamine:h2i interaction and the enhanced unwinding rate observed for $M t$ MCM Q181A are mutually consistent with an interaction that restricts interdomain movement and thus regulates helicase activity [60]. When the interaction is removed by mutation of the glutamine to alanine, the helicase activity is no longer regulated and can occur at an increased rate.

Additional roles for the ACL in the regulation of MCM catalyzed helicase activity are found in the observation that MtMCM E182R mutation results in a 7-fold decrease in unwinding rate relative to wild-type $M t \mathrm{MCM}$ for unwinding of forked DNA substrates [60]. In the Sso-PfMCM structure, the homologous glutamate residue (Sso-PfMCM E199) interacts with an arginine residue of an adjacent subunit, Sso-PfMCM R226 [44] (Figure 4(c)). Thus, the MtMCM E182R mutation would disrupt a salt bridge between adjacent subunits and likely increase the distance between subunits by electrostatic repulsion. Furthermore, the ACL is observed to project towards the AAA + psl $\beta$ motif of an adjacent subunit, which could be disrupted in the MtMCM E182R mutant due to an altered intersubunit distance. The proximity of the ACL to the h2i of the same subunit and the ps1 $\beta$ of a neighboring subunit has previously been shown by Double Electron-Electron Resonance (DEER) spectroscopy, with the ACL:ps1 $\beta$ distance estimated to be approximately $30 \AA$ [59]. While the role of the ACL:ps1 $\beta$ interaction is not clear, it may function like the ACL glutamine:h2i interaction to stabilize the ps1 $\beta$ and regulate MCM helicase activity. Thus, multiple roles have emerged for the ACL, including regulation of helicase activity, maintenance of intersubunit interfaces, and intrasubunit interactions.

\section{Concluding Comments}

Much like any machine, the MCM helicase requires specific events to occur in a precise order to unwind dsDNA. The identification of the essential events and their timing is complicated in the eukaryotic $\mathrm{Mcm} 2-7$ complex because the subunits do not equivalently interact, and they also interact with Cdc45 and the subunits of the GINS complex. For this reason, archaeal MCM proteins have emerged as useful models for elucidating the essential features of the complex interaction network present in MCM hexamers. A thorough understanding of the many interactions within the replisome is essential to understand the replication fork and DNA replication. Atomic resolution crystal structures will continue to reveal mechanistically important conformational states of the MCM complex, including how MCM hexamers specifically respond to binding different nucleotides, oligonucleotides, and protein interaction partners. As new information becomes available, both for the structural/biochemical details of MCM function and for disease-associated mutations, the translation of basic science information will undoubtedly aid in the treatment of primary disease states.

\section{Conflict of Interests}

The authors declare that there is no conflict of interests regarding the publication of this paper.

\section{Acknowledgments}

This work was supported in part by ALSAC and Grant R01GM098771 (to Eric J. Enemark) from NIGMS.

\section{References}

[1] M. L. Bochman and A. Schwacha, "The Mcm2-7 complex has in vitro helicase activity," Molecular Cell, vol. 31, no. 2, pp. 287-293, 2008.

[2] I. Ilves, T. Petojevic, J. J. Pesavento, and M. R. Botchan, "Activation of the MCM2-7 helicase by association with Cdc45 and GINS proteins," Molecular Cell, vol. 37, no. 2, pp. 247-258, 2010.

[3] Y. V. Fu, H. Yardimci, D. T. Long et al., "Selective bypass of a lagging strand roadblock by the eukaryotic replicative DNA helicase," Cell, vol. 146, no. 6, pp. 931-941, 2011.

[4] D. Remus, F. Beuron, G. Tolun, J. D. Griffith, E. P. Morris, and J. F. X. Diffley, "Concerted loading of Mcm2-7 double hexamers around DNA during DNA replication origin licensing," Cell, vol. 139, no. 4, pp. 719-730, 2009.

[5] C. Evrin, P. Clarke, J. Zech et al., "A double-hexameric MCM2-7 complex is loaded onto origin DNA during licensing of eukaryotic DNA replication," Proceedings of the National Academy of Sciences of the United States of America, vol. 106, no. 48, pp. 20240-20245, 2009.

[6] T. Masuda, S. Mimura, and H. Takisawa, "CDK- and Cdc45dependent priming of the MCM complex on chromatin during S-phase in Xenopus egg extracts: possible activation of MCM helicase by association with Cdc45," Genes to Cells, vol. 8, no. 2, pp. 145-161, 2003.

[7] J. A. Tercero, K. Labib, and J. F. X. Diffley, "DNA synthesis at individual replication forks requires the essential initiation factor Cdc45p," The EMBO Journal, vol. 19, no. 9, pp. 2082-2093, 2000 . 
[8] B. Hopwood and S. Dalton, "Cdc45p assembles into a complex with Cdc46p/Mcm5p, is required for minichromosome maintenance, and is essential for chromosomal DNA replication," Proceedings of the National Academy of Sciences of the United States of America, vol. 93, no. 22, pp. 12309-12314, 1996.

[9] Y. Kubota, Y. Takase, Y. Komori et al., "A novel ring-like complex of Xenopus proteins essential for the initiation of DNA replication," Genes and Development, vol. 17, no. 9, pp. 1141-1152, 2003.

[10] A. Gambus, R. C. Jones, A. Sanchez-Diaz et al., "GINS maintains association of $\mathrm{Cdc} 45$ with $\mathrm{MCM}$ in replisome progression complexes at eukaryotic DNA replication forks," Nature Cell Biology, vol. 8, no. 4, pp. 358-366, 2006.

[11] K. Labib and A. Gambus, "A key role for the GINS complex at DNA replication forks," Trends in Cell Biology, vol. 17, no. 6, pp. 271-278, 2007.

[12] S. E. Moyer, P. W. Lewis, and M. R. Botchan, "Isolation of the Cdc45/Mcm2-7/GINS (CMG) complex, a candidate for the eukaryotic DNA replication fork helicase," Proceedings of the National Academy of Sciences of the United States of America, vol. 103, no. 27, pp. 10236-10241, 2006.

[13] T. Yoshimochi, R. Fujikane, M. Kawanami, F. Matsunaga, and Y. Ishino, "The GINS complex from Pyrococcus furiosus stimulates the MCM helicase activity," The Journal of Biological Chemistry, vol. 283, no. 3, pp. 1601-1609, 2008.

[14] H. Yardimci, A. B. Loveland, S. Habuchi, A. M. van Oijen, and J. C. Walter, "Uncoupling of sister replisomes during eukaryotic DNA replication," Molecular Cell, vol. 40, no. 5, pp. 834-840, 2010.

[15] G. T. Haugland, C. R. Rollor, N.-K. Birkeland, and Z. Kelman, "Biochemical characterization of the minichromosome maintenance protein from the archaeon Thermoplasma acidophilum," Extremophiles, vol. 13, no. 1, pp. 81-88, 2009.

[16] Y. Ishimi, "A DNA helicase activity is associated with an MCM4, -6 , and -7 protein complex," The Journal of Biological Chemistry, vol. 272, no. 39, pp. 24508-24513, 1997.

[17] Z. Kelman, J.-K. Lee, and J. Hurwitz, "The single minichromosome maintenance protein of Methanobacterium thermoautotrophicum $\Delta \mathrm{H}$ contains DNA helicase activity," Proceedings of the National Academy of Sciences of the United States of America, vol. 96, no. 26, pp. 14783-14788, 1999.

[18] J.-K. Lee and J. Hurwitz, "Isolation and characterization of various complexes of the minichromosome maintenance proteins of Schizosaccharomyces pombe," The Journal of Biological Chemistry, vol. 275, no. 25, pp. 18871-18878, 2000.

[19] G. T. Maine, P. Sinha, and B. W. Tye, "Mutants of S. cerevisiae defective in the maintenance of minichromosomes," Genetics, vol. 106, no. 3, pp. 365-385, 1984.

[20] E. V. Koonin, "A common set of conserved motifs in a vast variety of putative nucleic acid-dependent ATPases including MCM proteins involved in the initiation of eukaryotic DNA replication," Nucleic Acids Research, vol. 21, no. 11, pp. 2541-2547, 1993.

[21] M. Lutzmann, D. Maiorano, and M. Méchali, "Identification of full genes and proteins of MCM9, a novel, vertebrate-specific member of the MCM2-8 protein family," Gene, vol. 362, no. 1-2, pp. 51-56, 2005.

[22] M. L. Bochman and A. Schwacha, "Differences in the singlestranded DNA binding activities of MCM2-7 and MCM467: MCM2 and MCM5 define a slow ATP-dependent step," The Journal of Biological Chemistry, vol. 282, no. 46, pp. 3379533804, 2007.
[23] A. Costa, I. Ilves, N. Tamberg et al., "The structural basis for MCM2-7 helicase activation by GINS and Cdc45," Nature Structural and Molecular Biology, vol. 18, no. 4, pp. 471-479, 2011.

[24] A. Costa, L. Renault, P. Swuec et al., "DNA binding polarity, dimerization, and ATPase ring remodeling in the CMG helicase of the eukaryotic replisome," Elife, vol. 3, Article ID e03273, 2014.

[25] J. P. J. Chong, M. K. Hayashi, M. N. Simon, R.-M. Xu, and B. Stillman, "A double-hexamer archaeal minichromosome maintenance protein is an ATP- dependent DNA helicase," Proceedings of the National Academy of Sciences of the United States of America, vol. 97, no. 4, pp. 1530-1535, 2000.

[26] D. F. Shechter, C. Y. Ying, and J. Gautier, "The intrinsic DNA helicase activity of Methanobacterium thermoautotrophicum delta $\mathrm{H}$ minichromosome maintenance protein," The Journal of Biological Chemistry, vol. 275, no. 20, pp. 15049-15059, 2000.

[27] F. Sarmiento, F. Long, I. Cann, and W. B. Whitman, "Diversity of the DNA replication system in the archaea domain," Archaea, vol. 2014, Article ID 675946, 15 pages, 2014.

[28] A. Costa, G. Van Duinen, B. Medagli et al., "Cryo-electron microscopy reveals a novel DNA-binding site on the MCM helicase," The EMBO Journal, vol. 27, no. 16, pp. 2250-2258, 2008.

[29] X. Yu, M. S. VanLoock, A. Poplawski et al., "The Methanobacterium thermoautotrophicum MCM protein can form heptameric rings," EMBO Reports, vol. 3, no. 8, pp. 792-797, 2002.

[30] T. Pape, H. Meka, S. Chen, G. Vicentini, M. van Heel, and S. Onesti, "Hexameric ring structure of the full-length archael MCM protein complex," EMBO Reports, vol. 4, no. 11, pp. 10791083, 2003.

[31] Y. Gómez-Llorente, R. J. Fletcher, X. S. Chen, J. M. Carazo, and C. S. Martin, "Polymorphism and double hexamer structure in the archaeal minichromosome maintenance (MCM) helicase from Methanobacterium thermoautotrophicum," The Journal of Biological Chemistry, vol. 280, no. 49, pp. 40909-40915, 2005.

[32] E. R. Barry, A. T. McGeoch, Z. Kelman, and S. D. Bell, "Archaeal MCM has separable processivity, substrate choice and helicase domains," Nucleic Acids Research, vol. 35, no. 3, pp. 988-998, 2007.

[33] A. F. Neuwald, L. Aravind, J. L. Spouge, and E. V. Koonin, "AAA+: a class of chaperone-like ATPases associated with the assembly, operation, and disassembly of protein complexes," Genome Research, vol. 9, no. 1, pp. 27-43, 1999.

[34] T. Ogura and A. J. Wilkinson, "AAA ${ }^{+}$superfamily ATPases: common structure-diverse function," Genes to Cells, vol. 6, no. 7, pp. 575-597, 2001.

[35] L. M. Iyer, D. D. Leipe, E. V. Koonin, and L. Aravind, "Evolutionary history and higher order classification of AAA+ ATPases," Journal of Structural Biology, vol. 146, no. 1-2, pp. 11-31, 2004.

[36] P. I. Hanson and S. W. Whiteheart, "AAA+ proteins: have engine, will work," Nature Reviews Molecular Cell Biology, vol. 6, no. 7, pp. 519-529, 2005.

[37] L. Aravind and E. V. Koonin, "DNA-binding proteins and evolution of transcription regulation in the archaea," Nucleic Acids Research, vol. 27, no. 23, pp. 4658-4670, 1999.

[38] N. Atanassova and I. Grainge, "Biochemical characterization of the minichromosome maintenance (MCM) protein of the crenarchaeote Aeropyrum pernix and its interactions with the origin recognition complex (ORC) proteins," Biochemistry, vol. 47, no. 50, pp. 13362-13370, 2008. 
[39] R. J. Fletcher, B. E. Bishop, R. P. Leon, R. A. Sclafani, C. M. Ogata, and X. S. Chen, "The structure and function of MCM from archaeal M. thermoautotrophicum," Nature Structural Biology, vol. 10, no. 3, pp. 160-167, 2003.

[40] W. Liu, B. Pucci, M. Rossi, F. M. Pisani, and R. Ladenstein, "Structural analysis of the Sulfolobus solfataricus MCM protein N-terminal domain," Nucleic Acids Research, vol. 36, no. 10, pp. 3235-3243, 2008.

[41] A. S. Brewster, G. Wang, X. Yu et al., "Crystal structure of a nearfull-length archaeal MCM: functional insights for an AAA+ hexameric helicase," Proceedings of the National Academy of Sciences of the United States of America, vol. 105, no. 51, pp. 20191-20196, 2008.

[42] B. Bae, Y. H. Chen, A. Costa et al., "Insights into the architecture of the replicative helicase from the structure of an archaeal MCM homolog," Structure, vol. 17, no. 2, pp. 211-222, 2009.

[43] C. A. Froelich, S. Kang, L. B. Epling, S. P. Bell, and E. J. Enemark, "A conserved MCM single-stranded DNA binding element is essential for replication initiation," eLife, vol. 2014, no. 3, Article ID e01993, 2014.

[44] J. M. Miller, B. T. Arachea, L. B. Epling, and E. J. Enemark, "Analysis of the crystal structure of an active MCM hexamer," eLife, vol. 3, Article ID e03433, 2014.

[45] Y.-J. Chen, X. Yu, R. Kasiviswanathan, J.-H. Shin, Z. Kelman, and E. H. Egelman, "Structural polymorphism of Methanothermobacter thermautotrophicus MCM," Journal of Molecular Biology, vol. 346, no. 2, pp. 389-394, 2005.

[46] R. J. Fletcher and X. S. Chen, "Biochemical activities of the BOB1 mutant in Methanobacterium thermoautotrophicum MCM," Biochemistry, vol. 45, no. 2, pp. 462-467, 2006.

[47] S. Krueger, J.-H. Shin, S. Raghunandan, J. E. Curtis, and Z. Kelman, "Atomistic ensemble modeling and small-angle neutron scattering of intrinsically disordered protein complexes: applied to minichromosome maintenance protein," Biophysical Journal, vol. 101, no. 12, pp. 2999-3007, 2011.

[48] S. Krueger, J.-H. Shin, J. E. Curtis, K. A. Rubinson, and Z. Kelman, "The solution structure of full-length dodecameric MCM by SANS and molecular modeling," Proteins, vol. 82, no. 10, pp. 2364-2374, 2014.

[49] R. Kasiviswanathan, J.-H. Shin, E. Melamud, and Z. Kelman, "Biochemical characterization of the Methanothermobacter thermautotrophicus minichromosome maintenance (MCM) helicase N-terminal domains," The Journal of Biological Chemistry, vol. 279, no. 27, pp. 28358-28366, 2004.

[50] B. W. Graham, G. D. Schauer, S. H. Leuba, and M. A. Trakselis, "Steric exclusion and wrapping of the excluded DNA strand occurs along discrete external binding paths during MCM helicase unwinding," Nucleic Acids Research, vol. 39, no. 15, pp. 6585-6595, 2011.

[51] T. Petojevic, J. J. Pesavento, A. Costa et al., "Cdc45 (cell division cycle protein 45) guards the gate of the Eukaryote Replisome helicase stabilizing leading strand engagement," Proceedings of the National Academy of Sciences, vol. 112, no. 3, pp. E249-E258, 2015.

[52] E. Rothenberg, M. A. Trakselis, S. D. Bell, and T. Ha, "MCM forked substrate specificity involves dynamic interaction with the $5^{\prime}$-tail," Journal of Biological Chemistry, vol. 282, no. 47, pp. 34229-34234, 2007.

[53] C. F. S. Hardy, O. Dryga, S. Seematter, P. M. B. Pahl, and R. A. Sclafani, "mcm5/cdc46-bob1 bypasses the requirement for the $S$ phase activator Cdc7p," Proceedings of the National Academy of
Sciences of the United States of America, vol. 94, no. 7, pp. 31513155, 1997.

[54] E. R. Jenkinson, A. Costa, A. P. Leech, A. Patwardhan, S. Onesti, and J. P. J. Chong, "Mutations in subdomain B of the minichromosome maintenance (MCM) helicase affect DNA binding and modulate conformational transitions," Journal of Biological Chemistry, vol. 284, no. 9, pp. 5654-5661, 2009.

[55] A. Poplawski, B. Grabowski, S. E. Long, and Z. Kelman, "The zinc finger domain of the archaeal minichromosome maintenance protein is required for helicase activity," The Journal of Biological Chemistry, vol. 276, no. 52, pp. 49371-49377, 2001.

[56] B. K. Tye, "MCM proteins in DNA replication," Annual Review of Biochemistry, vol. 68, pp. 649-686, 1999.

[57] A. G. Murzin, “OB(oligonucleotide/oligosaccharide binding)fold: common structural and functional solution for nonhomologous sequences," The EMBO Journal, vol. 12, no. 3, pp. 861-867, 1993.

[58] N. Sakakibara, R. Kasiviswanathan, and Z. Kelman, "Mutational analysis of conserved aspartic acid residues in the Methanothermobacter thermautotrophicus MCM helicase," Extremophiles, vol. 15, no. 2, pp. 245-252, 2011.

[59] E. R. Barry, J. E. Lovett, A. Costa, S. M. Lea, and S. D. Bell, "Intersubunit allosteric communication mediated by a conserved loop in the MCM helicase," Proceedings of the National Academy of Sciences of the United States of America, vol. 106, no. 4, pp. 1051-1056, 2009.

[60] N. Sakakibara, R. Kasiviswanathan, E. Melamud, M. Han, F. P. Schwarz, and Z. Kelman, "Coupling of DNA binding and helicase activity is mediated by a conserved loop in the MCM protein," Nucleic Acids Research, vol. 36, no. 4, pp. 1309-1320, 2008.

[61] N. Shima, A. Alcaraz, I. Liachko et al., "A viable allele of Mcm4 causes chromosome instability and mammary adenocarcinomas in mice," Nature Genetics, vol. 39, no. 1, pp. 93-98, 2007.

[62] V. G. Gorgoulis, L.-V. F. Vassiliou, P. Karakaidos et al., "Activation of the DNA damage checkpoint and genomic instability in human precancerous lesions," Nature, vol. 434, no. 7035, pp. 907-913, 2005.

[63] C. Lengauer, K. W. Kinzler, and B. Vogelstein, "Genetic instabilities in human cancers," Nature, vol. 396, no. 6712, pp. 643-649, 1998.

[64] S. Negrini, V. G. Gorgoulis, and T. D. Halazonetis, "Genomic instability-an evolving hallmark of cancer," Nature Reviews Molecular Cell Biology, vol. 11, no. 3, pp. 220-228, 2010.

[65] M. J. Davey, C. Indiani, and M. O’Donnell, "Reconstitution of the Mcm2-7p heterohexamer, subunit arrangement, and ATP site architecture," The Journal of Biological Chemistry, vol. 278, no. 7, pp. 4491-4499, 2003.

[66] M. J. Moreau, A. T. McGeoch, A. R. Lowe, L. S. Itzhaki, and S. D. Bell, "ATPase site architecture and helicase mechanism of an archaeal MCM," Molecular Cell, vol. 28, no. 2, pp. 304-314, 2007.

[67] N. Sakakibara, F. P. Schwarz, and Z. Kelman, "ATP hydrolysis and DNA binding confer thermostability on the MCM helicase," Biochemistry, vol. 48, no. 11, pp. 2330-2339, 2009.

[68] Z. Wei, C. Liu, X. Wu et al., "Characterization and structure determination of the cdt1 binding domain of human minichromosome maintenance (Mcm) 6," The Journal of Biological Chemistry, vol. 285, no. 17, pp. 12469-12473, 2010.

[69] P. D. Robertson, B. Chagot, W. J. Chazin, and B. F. Eichman, "Solution NMR structure of the C-terminal DNA binding domain of Mcm10 reveals a conserved MCM motif," The Journal of Biological Chemistry, vol. 285, no. 30, pp. 22942-22949, 2010. 
[70] P. D. Robertson, E. M. Warren, H. Zhang et al., "Domain architecture and biochemical characterization of vertebrate Mcm10," The Journal of Biological Chemistry, vol. 283, no. 6, pp. 3338-3348, 2008.

[71] C. Wiedemann, P. Bellstedt, C. Herbst, M. Görlach, and R. Ramachandran, "An approach to sequential NMR assignments of proteins: application to chemical shift restraint-based structure prediction," Journal of Biomolecular NMR, vol. 59, no. 4, pp. 211-217, 2014.

[72] E. R. Jenkinson and J. P. J. Chong, "Minichromosome maintenance helicase activity is controlled by $\mathrm{N}$ - and C-terminal motifs and requires the ATPase domain helix-2 insert," Proceedings of the National Academy of Sciences of the United States of America, vol. 103, no. 20, pp. 7613-7618, 2006.

[73] A. T. McGeoch, M. A. Trakselis, R. A. Laskey, and S. D. Bell, "Organization of the archaeal MCM complex on DNA and implications for the helicase mechanism," Nature Structural and Molecular Biology, vol. 12, no. 9, pp. 756-762, 2005.

[74] B. Pucci, M. De Felice, M. Rossi, S. Onesti, and F. M. Pisani, "Amino acids of the Sulfolobus solfataricus minichromosome maintenance-like DNA helicase involved in DNA binding/remodeling," Journal of Biological Chemistry, vol. 279, no. 47, pp. 49222-49228, 2004.

[75] M. Pan, T. J. Santangelo, Z. Li, J. N. Reeve, and Z. Kelman, "Thermococcus kodakarensis encodes three MCM homologs but only one is essential," Nucleic Acids Research, vol. 39, no. 22, pp. 9671-9680, 2011.

[76] X. Zhang and D. B. Wigley, “The 'glutamate switch' provides a link between ATPase activity and ligand binding in AAA+ proteins," Nature Structural and Molecular Biology, vol. 15, no. 11, pp. 1223-1227, 2008.

[77] E. J. Enemark and L. Joshua-Tor, "Mechanism of DNA translocation in a replicative hexameric helicase," Nature, vol. 442, no. 7100, pp. 270-275, 2006.

[78] D. Gai, R. Zhao, D. Li, C. V. Finkielstein, and X. S. Chen, "Mechanisms of conformational change for a replicative hexameric helicase of SV40 large tumor antigen," Cell, vol. 119, no. 1, pp. 47-60, 2004.

[79] J. P. Erzberger and J. M. Berger, "Evolutionary relationships and structural mechanisms of AAA+ proteins," Annual Review of Biophysics and Biomolecular Structure, vol. 35, no. 1, pp. 93-114, 2006.

[80] R. J. Fletcher, J. Shen, Y. Gómez-Llorente, C. San Martín, J. M. Carazo, and X. S. Chen, "Double hexamer disruption and biochemical activities of Methanobacterium thermoautotrophicum MCM," The Journal of Biological Chemistry, vol. 280, no. 51, pp. 42405-42410, 2005.

[81] R. A. Sclafani, R. J. Fletcher, and X. S. Chen, "Two heads are better than one: regulation of DNA replication by hexameric helicases," Genes and Development, vol. 18, no. 17, pp. 2039-2045, 2004.

[82] O. Itsathitphaisarn, R. A. Wing, W. K. Eliason, J. Wang, and T. A. Steitz, "The hexameric helicase DnaB adopts a nonplanar conformation during translocation," Cell, vol. 151, no. 2, pp. 267277, 2012.

[83] N. D. Thomsen and J. M. Berger, "Running in reverse: the structural basis for translocation polarity in hexameric helicases," Cell, vol. 139, no. 3, pp. 523-534, 2009.

[84] R. P. Leon, M. Tecklenburg, and R. A. Sclafani, "Functional conservation of $\beta$-hairpin DNA binding domains in the $\mathrm{Mcm}$ protein of Methanobacterium thermoautotrophicum and the
Mcm5 protein of Saccharomyces cerevisiae," Genetics, vol. 179, no. 4, pp. 1757-1768, 2008.

[85] N. D. Thomsen and J. M. Berger, "Structural frameworks for considering microbial protein- and nucleic acid-dependent motor ATPases," Molecular Microbiology, vol. 69, no. 5, pp. 10711090, 2008.

[86] Z. Yu, D. Feng, and C. Liang, "Pairwise interactions of the six human MCM protein subunits," Journal of Molecular Biology, vol. 340, no. 5, pp. 1197-1206, 2004.

[87] E. F. Pettersen, T. D. Goddard, C. C. Huang et al., "UCSF Chimera-a visualization system for exploratory research and analysis," Journal of Computational Chemistry, vol. 25, no. 13, pp. 1605-1612, 2004.

[88] The PyMOL Molecular Graphics System, Version 1.5.0.4 Schrödinger, LLC.

[89] J. M. Berger, "SnapShot: nucleic acid helicases and translocases," Cell, vol. 134, no. 5, pp. 888-888.e1, 2008. 

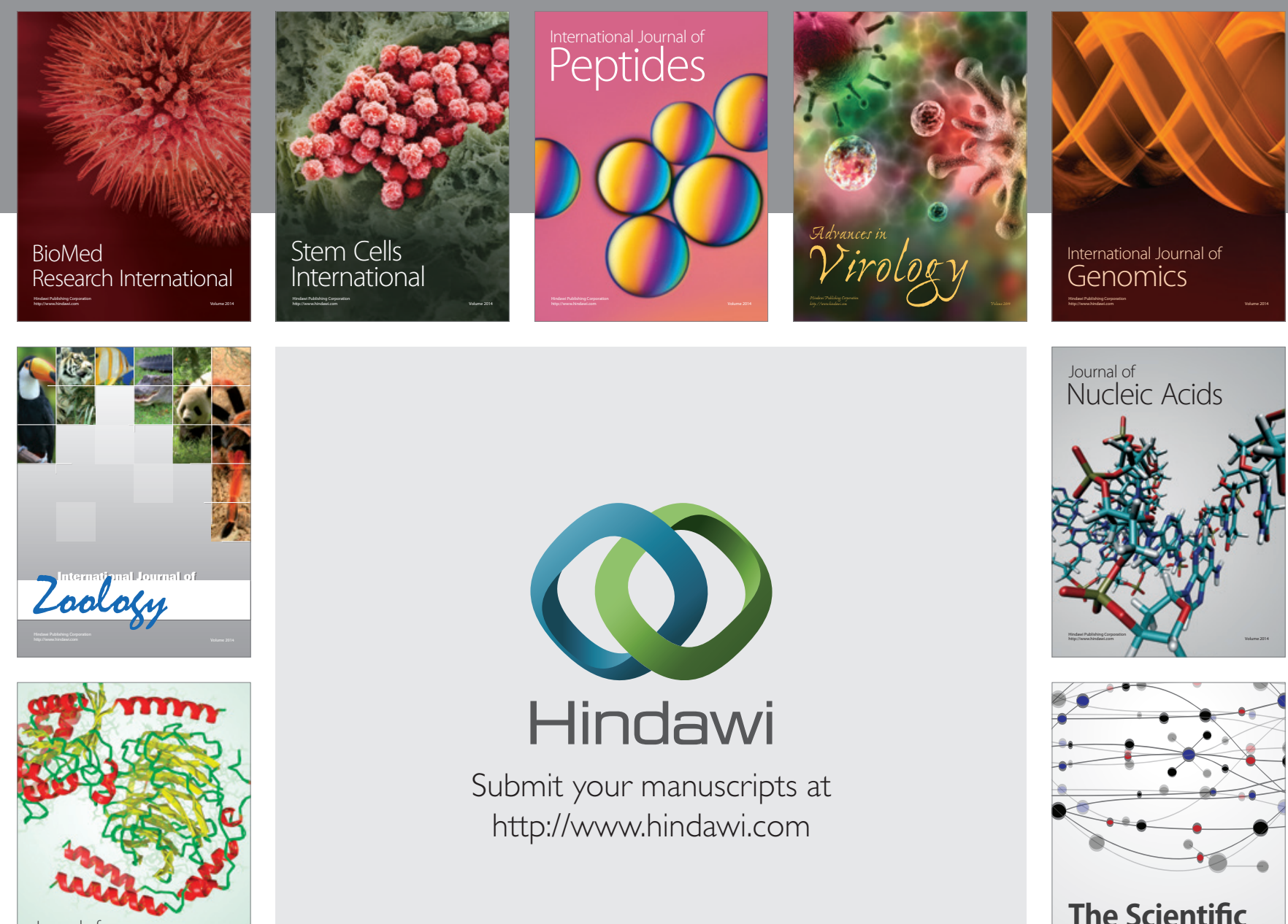

Submit your manuscripts at

http://www.hindawi.com

Journal of
Signal Transduction
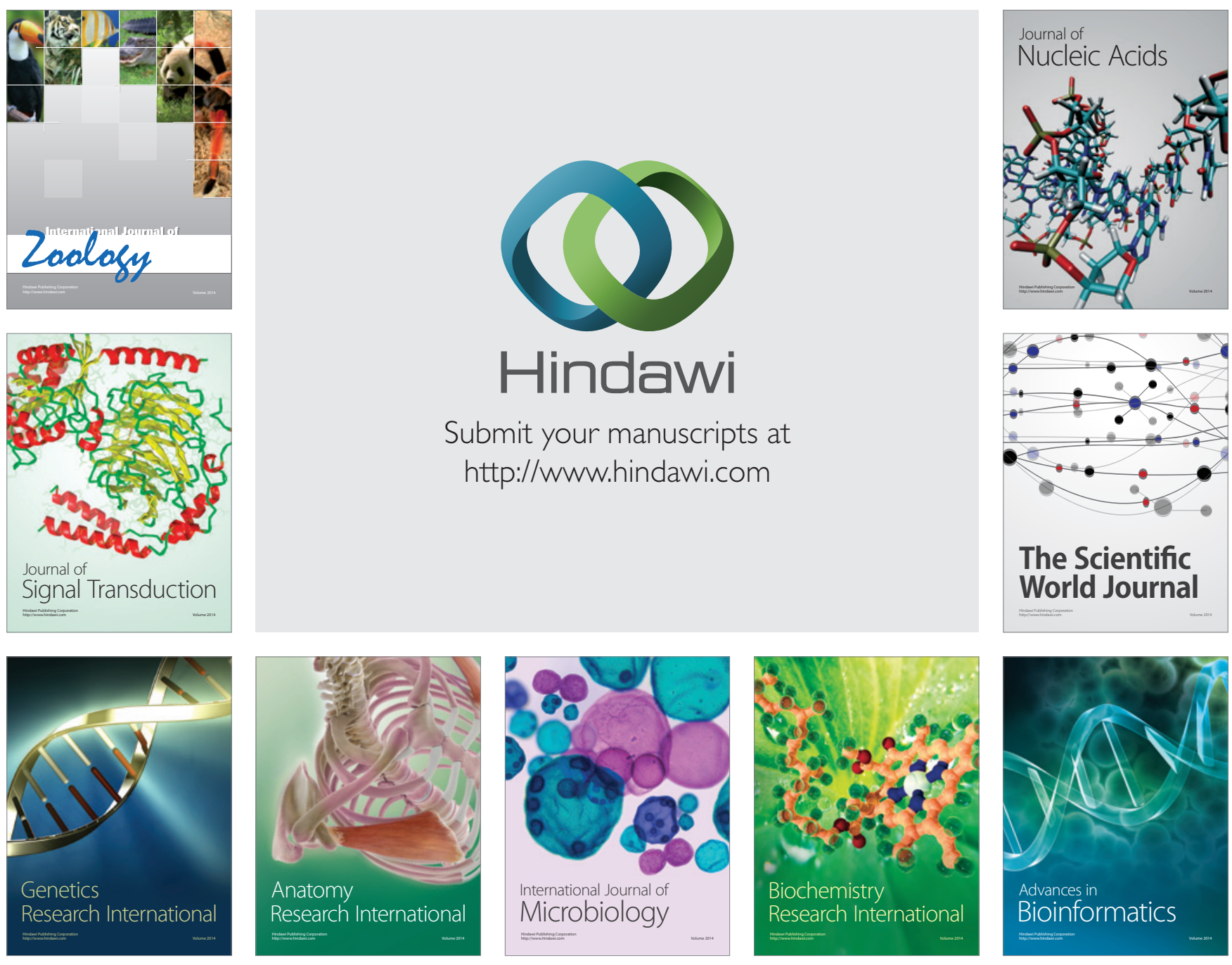

The Scientific World Journal
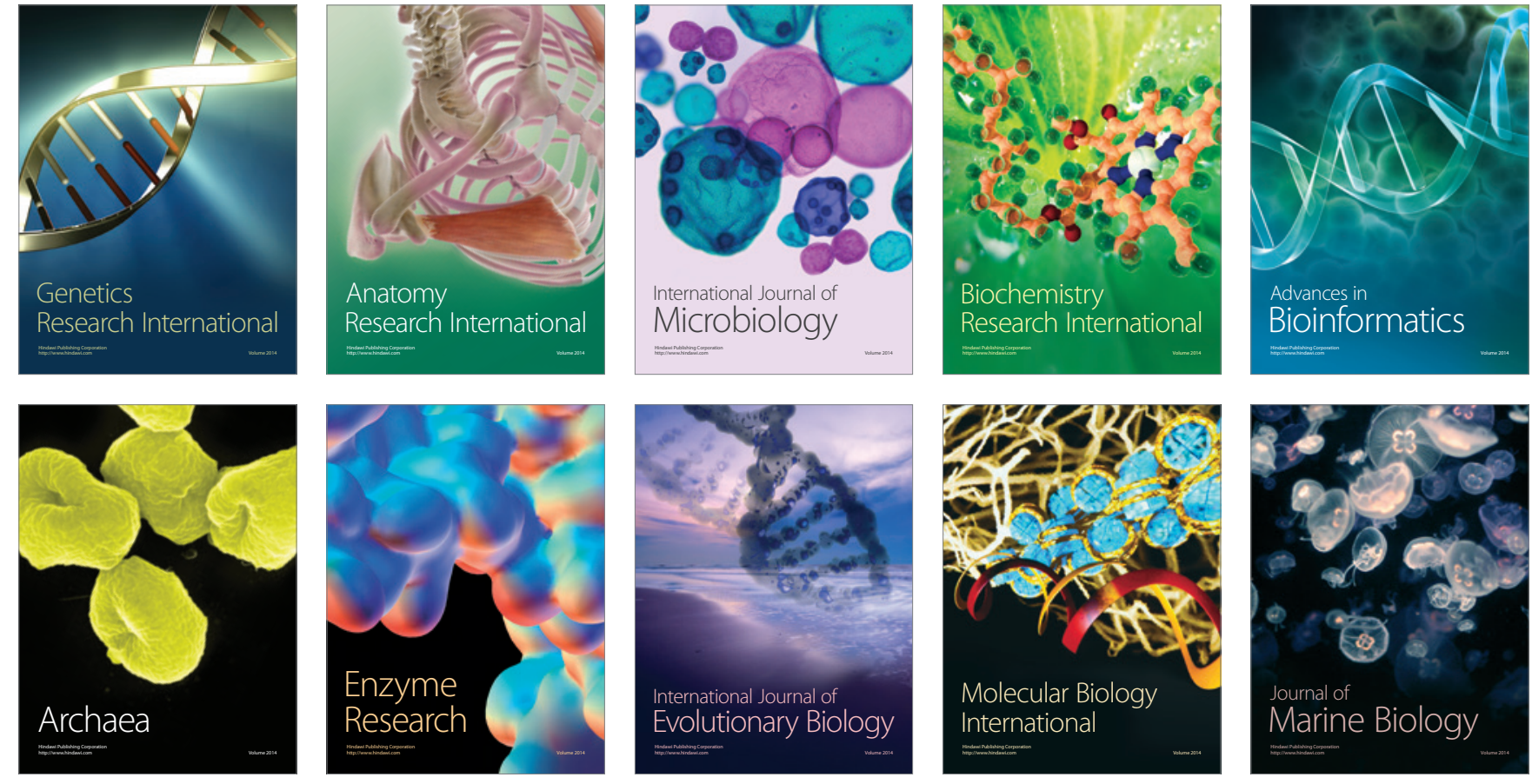